\title{
The impact of metallicity-dependent dust destruction on the dust-to-metals ratio in galaxies
}

\author{
F. D. Priestley ${ }^{1 \star}$, I. De Looze ${ }^{2,3}$ and M. J. Barlow ${ }^{3}$ \\ ${ }^{1}$ School of Physics and Astronomy, Cardiff University, Queen's Buildings, The Parade, Cardiff CF24 3AA, UK \\ ${ }^{2}$ Sterrenkundig Observatorium, Ghent University, Krijgslaan 281 - S9, 9000 Gent, Belgium \\ ${ }^{3}$ Department of Physics and Astronomy, University College London, Gower Street, London WC1E 6BT, UK
}

Accepted XXX. Received YYY; in original form ZZZ

\begin{abstract}
The ratio of the mass of interstellar dust to the total mass of metals (the dust-to-metals/DTM ratio) tends to increase with metallicity. This can be explained by the increasing efficiency of grain growth in the interstellar medium (ISM) at higher metallicities, with a corollary being that the low DTM ratios seen at low metallicities are due to inefficient stellar dust production. This interpretation assumes that the efficiency of dust destruction in the ISM is constant, whereas it might be expected to increase at low metallicity; the decreased cooling efficiency of low-metallicity gas should result in more post-shock dust destruction via thermal sputtering. We show that incorporating a sufficiently strong metallicity dependence into models of galaxy evolution removes the/need for low stellar dust yields. The contribution of stellar sources to the overall dust budget may be significantly underestimated, and that of grain growth overestimated, by models assuming a constant destruction efficiency.
\end{abstract}

Key words:

dust, extinction - ISM: evolution

\section{INTRODUCTION}

The variation of the dust-to-metals (DTM) ratio with the total metal content of galaxies is often seen as strong evidence for grain growth in the interstellar medium (ISM) as the main driver of dust evolution. Models often struggle to reproduce the observed distribution of galaxies in the DTM ratio-metallicity plane with efficient dust production by core-collapse supernovae (CCSNe), leaving ISM growth as the only way to account for the observed dust masses (De Vis et al. 2017, 2019; Galliano et al. 2021) unless dust yields from lower-mass stars are substantially higher than is typically assumed. However, the required limits on CCSN dust yields ( $\lesssim 0.1 \mathrm{M}_{\odot}$ per CCSN) are well below those observed in supernova remnants (Matsuura et al. 2015; De Looze et al 2017, 2019; Chawner et al. 2019), even after accounting for possible future destruction via reverse shocks (De Looze et al. 2017; Priestley et al. 2019; Niculescu-Duvaz et al. 2021).

While models of ISM enrichment and evolution typically account for the metallicity-dependence of grain growth, in that the rate of accretion increases with the availability of gas-phase metals, the efficiency of dust destruction in the

^ Email: priestleyf@cardiff.ac.uk
ISM is often assumed to be constant. This is potentially inaccurate; metal-poor gas cools less efficiently, thus remaining at high temperatures for longer, and increasing the amount of dust destroyed by thermal sputtering in shocked gas (Yamasawa et al. 2011, hereafter Y11). Some studies of dust evolution (e.g. Triani et al. 2020) do use a metallicitydependent destruction efficiency, but to our knowledge, the impact of such an evolving efficiency has not been investigated in detail. In this paper, we show that an increasing destruction efficiency at low metallicity may remove the tension between high stellar dust yields and the low DTM ratios seen in some galaxies, if the dependence on metallicity is strong enough. Without accounting for this effect, the importance of stellar sources to the global dust budget may be significantly underestimated.

\section{METHOD}

We consider a closed-box model of galaxy evolution with a single-phase ISM, tracking four main quantities; the masses of gas $\left(M_{\text {gas }}\right)$, stars $\left(M_{*}\right)$, gas-phase metals $\left(M_{Z}\right)$, and dust $\left(M_{\text {dust }}\right)$. Initially-pristine gas is converted into stars, which return a fraction of that gas to the ISM, enriched with metals and dust under the instantaneous-recycling approximation. 
Table 1. Values of model parameters which are held constant.

\begin{tabular}{ccc}
\hline Parameter & Value & Unit \\
\hline$f_{\text {ret }}$ & 0.1 & - \\
$y_{Z}+y_{\text {dust }}$ & 0.1 & - \\
$A_{g}$ & $4.24 \times 10^{5}$ & $\mathrm{~cm}^{2} \mathrm{~g}^{-1}$ \\
$T_{\text {gas }}$ & 20 & $\mathrm{~K}$ \\
$\mu_{Z}$ & 12 & $m_{\mathrm{H}}$ \\
$\mu$ & 2.33 & $m_{\mathrm{H}}$ \\
$n_{\mathrm{H}}$ & 100 & $\mathrm{~cm}^{-3}$ \\
$f_{\mathrm{SN}}$ & 0.01 & $\mathrm{M}_{\odot}^{-1}$ \\
\hline
\end{tabular}

Gas-phase metals are accreted onto dust grains, and metals locked up in dust grains are released into the gas via dust destruction. The evolution of the system is given by

$$
\begin{array}{r}
\frac{d M_{\text {gas }}}{d t}=-\left(1-f_{\text {ret }}\right) \Sigma \\
\frac{d M_{*}}{d t}=\left(1-f_{\text {ret }}\right) \Sigma \\
\frac{d M_{Z}}{d t}=\left(f_{\text {ret }} y_{Z}-Z_{\text {gas }}\right) \Sigma+D-G \\
\frac{d M_{\text {dust }}}{d t}=\left(f_{\text {ret }} y_{\text {dust }}-Z_{\text {dust }}\right) \Sigma-D+G
\end{array}
$$

where $\Sigma$ is the star formation rate (SFR), $f_{\text {ret }}$ is the fraction of mass returned by stars into the ISM, $y_{Z}$ and $y_{\text {dust }}$ are the stellar yields of gas-phase metals and dust respectively (the fraction of mass returned in the form of either), $Z_{\text {gas }}=$ $M_{Z} / M_{\text {gas }}$ and $Z_{\text {dust }}=M_{\text {dust }} / M_{\text {gas }}$ are the gas- and dustphase metallicities, and $D$ and $G$ are the dust destruction and growth rates respectively. The total metallicity of the 'galaxy' is $Z_{\text {tot }}=\left(M_{Z}+M_{\text {dust }}\right) / M_{\text {gas }}=Z_{\text {gas }}+Z_{\text {dust }}$, and the DTM ratio is $M_{\text {dust }} /\left(M_{Z}+M_{\text {dust }}\right)$. We assume initial values of $M_{\text {gas }}=10^{10} \mathrm{M}_{\odot}$ and $M_{*}=M_{Z}=M_{\text {dust }}=0$, and evolve the model for $10 \mathrm{Gyr}$, with an adaptive timestep of $0.01 \times$ $\min \left(M_{\mathrm{gas}} / \Sigma, M_{Z} / G, M_{\mathrm{dust}} / D\right)$. As we are mainly interested in ratios of masses, rather than their absolute values, the initial value of $M_{\text {gas }}$ has no impact on our results. We take the Asplund et al. (2009) value of $\mathrm{Z}_{\odot}=0.0134$ for solar metallicity.

\subsection{Star formation}

The SFR, $\Sigma$, is not in general a function of the other model parameters, as galaxies grow and evolve due to the accretion and ejection of gas, and occasional mergers (Triani et al. 2020; Nanni et al. 2020). We assume

$\Sigma=2 \times 10^{6}\left(\frac{M_{\text {gas }}}{10^{10} M_{\odot}}\right) M_{\odot} \mathrm{Myr}^{-1}$,

which gives $1 \mathrm{M}_{\odot} \mathrm{yr}^{-1}$ for a gas mass of $5 \times 10^{9} \mathrm{M}_{\odot}$, in line with Milky Way values (Dame 1993; Robitaille \& Whitney 2010), and produces an exponentially-declining SFR. The galaxy scaling relations we are interested in are typically more sensitive to the assumptions made regarding the dust physics, rather than the star formation history (De Vis et al. 2017; De Looze et al. 2020).

\subsection{Grain growth}

The increase in dust mass due to growth in the ISM is given by

$G=f_{\text {acc }} \times A_{g} M_{\text {gas }} Z_{\text {dust }} \times \sqrt{\frac{k_{b} T_{\text {gas }}}{\mu_{Z}}} \mu n_{\mathrm{H}} Z_{\text {gas }}$.

Here, $A_{g}$ is the grain surface area per unit mass, $k_{b}$ is the Boltzmann constant, $T_{\text {gas }}, n_{\mathrm{H}}$ and $\mu$ the gas temperature, number density and mean molecular mass in the phase of the ISM where grain growth occurs, and $\mu_{Z}$ is the mass of a typical accreting atom. $f_{\text {acc }}$ is a parameter representing, among other things, the fraction of the total gas mass in which grain growth occurs, and the sticking efficiency of collisions between atoms and grains. The second term in Equation 6 gives the total available grain surface area for accretion, and the third term is the thermal velocity of accreting atoms multiplied by their mass density, giving the mass flux' per unit area.

\subsection{Dust destruction}

Along with astration and outflows, destruction in shocks driven by $\mathrm{SNe}$ is one of the main processes removing dust from the ISM. As individual SNe cannot be resolved even in hydrodynamical simulations of galaxy evolution, their effects are generally abstracted to a dust destruction timescale representing the typical lifetime of grains in the ISM (e.g. Morgan \& Edmunds 2003). We assume the rate of dust destruction is given by

$D=f_{\mathrm{SN}} \Sigma \times Z_{\text {dust }} M_{\text {clear }}$,

where $f_{\mathrm{SN}}$ is the number of SNe per unit mass of stars formed, $\Sigma$ is the SFR given in Equation 5 , and $M_{\text {clear }}$ is the gas mass 'cleared' of dust per SN.

\subsection{Parameter choices}

The free parameters controlling grain growth and destruction in Equations 6 and 7 are degenerate, so we fix all but one in each case to physically-reasonable values. For grain growth, we assume that this process occurs in molecular gas with $n_{\mathrm{H}}=100 \mathrm{~cm}^{-3}, T_{\text {gas }}=20 \mathrm{~K}$ and $\mu=2.33 m_{\mathrm{H}}$, that the accreting atoms have $\mu_{Z}=12 m_{\mathrm{H}}$, and that the area-to-mass ratio of dust grains is that of a Mathis et al. (1977) distribution with a bulk density of $2 \mathrm{~g} \mathrm{~cm}^{-3}\left(4.24 \times 10^{5} \mathrm{~cm}^{2} \mathrm{~g}^{-1}\right)$. For $f_{\mathrm{SN}}$, we assume a value of $0.01 \mathrm{M}_{\odot}^{-1}$ consistent with a Salpeter (1955) initial mass function and a minimum SN mass of $\sim 10 \mathrm{M}_{\odot}$. We additionally assume a returned mass fraction $f_{\text {ret }}=0.1$ and a total metal yield $y_{Z}+y_{\text {dust }}=0.1$, in line with stellar evolution models (Woosley \& Weaver 1995). The metal yields of both asymptotic giant branch (AGB) stars (Cristallo et al. 2009) and CCSNe from $\lesssim 30 \mathrm{M}_{\odot}$ progenitors ${ }^{1}$ (Limongi \& Chieffi 2018) appear to vary only modestly with metallicity, so we take these values as constant. Values of the fixed parameters are listed in Table 1.

1 Higher-mass progenitors account for only $\sim 20 \%$ of CCSNe for a Salpeter (1955) power law, so are relatively unimportant as a source of metals. 

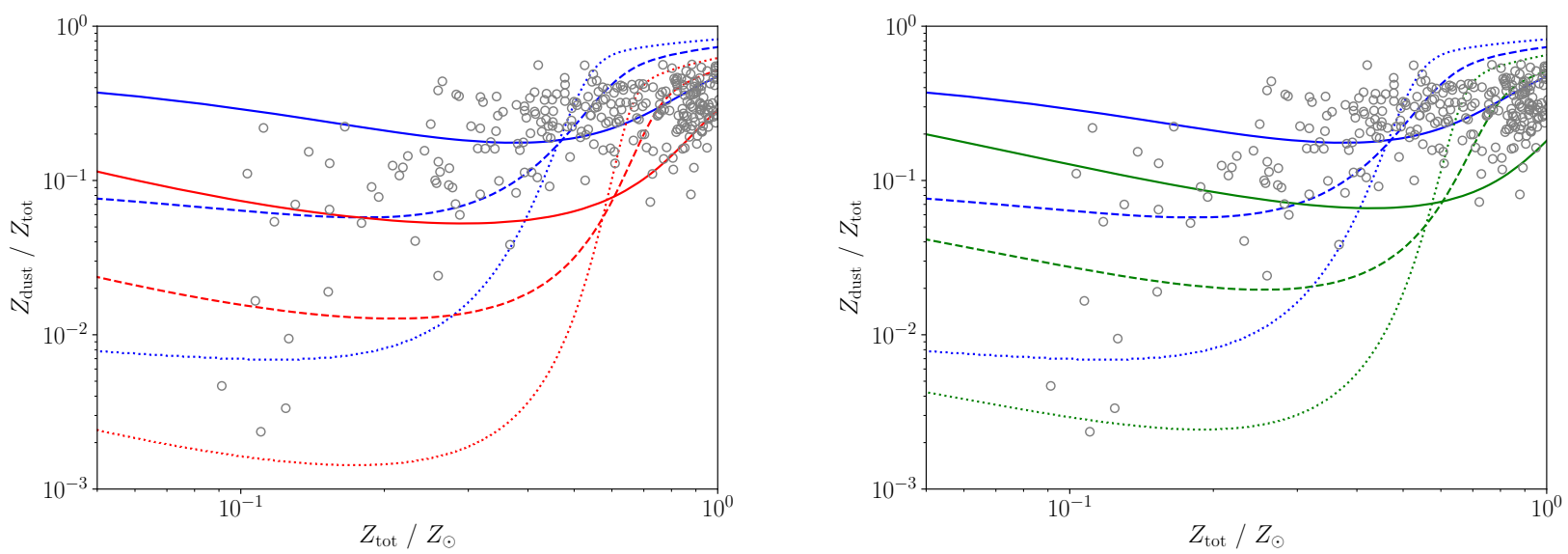

Figure 1. Evolution of the DTM ratio versus metallicity for models with a $y_{\text {dust }}\left(f_{\text {acc }}\right)$ of $0.05(0.005)$ (solid lines), 0.01 (0.01) (dashed lines) and 0.001 (0.015) (dotted lines). The left panel shows models with a constant $M_{\text {clear }}=1000 \mathrm{M}_{\odot}$ (blue lines) and with $M_{\text {clear }}$ given by Equation 8 (red lines). The right panel shows models with a constant $M_{\text {clear }}=1000 \mathrm{M}_{\odot}$ (blue lines) and with the Y11 $M_{\text {clear }}$ (green lines). Observational data from Galliano et al. (2021) are shown as grey open circles.

The remaining free parameters are the grain growth efficiency $f_{\text {acc }}$, the mass of gas cleared per SN $M_{\text {clear }}$, and the distribution of newly-produced metals between the gas and dust phases, $y_{Z}$ and $y_{\text {dust }}$. We assume that $f_{\text {acc }}$ and the balance between $y_{Z}$ and $y_{\text {dust }}$ both remain constant with metallicity. The accretion efficiency is not necessarily constant, as both the fraction of the accreting phase of the ISM (Mattsson et al. 2012) and the ability of grains to accrete atoms (Ferrara et al. 2016) may change over time, but our results are not greatly affected by introducing additional dependencies (e.g. $f_{\text {acc }} \propto \Sigma$ ). As with the total metal yields, theoretical DTM ratios of both AGB outflows (Nanni et al. 2013) and CCSN ejecta from lower-mass progenitors (Marassi et al. 2019) do not appear to vary significantly with metallicity.

The cleared gas mass per SN, $M_{\text {clear }}$, is typically estimated to be $\sim 1000 \mathrm{M}_{\odot}$ under local ISM conditions (Jones et al. 1994; Hu et al. 2019), although there are arguments in favour of a lower value (Martínez-González et al. 2019; Priestley et al. 2021; Ferrara \& Peroux 2021). $M_{\text {clear }}$ is also likely to vary with metallicity; at lower $Z_{\text {gas }}$, gas cools less efficiently, and thus remains at the high temperatures necessary for efficient thermal sputtering for longer. Based on the variation with metallicity of the dust destruction efficiency derived in Appendix A, we consider

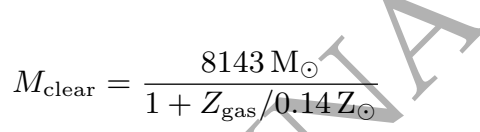

which gives $M_{\text {clear }} \sim 1000 \mathrm{M}_{\odot}$ at $Z_{\text {gas }}=\mathrm{Z}_{\odot}$. We also investigate módels with a constant $M_{\text {clear }}=1000 \mathrm{M}_{\odot}$ for comparison.

Equation 8 may overestimate the strength of the dependence of $M_{\text {clear }}$ on $Z_{\text {gas }}$ - Y11, using a more detailed model of dust destruction, find a much weaker scaling $\left(M_{\text {clear }} \sim\right.$ $Z^{-0.298}$ at high $Z$ ). We use their eq. 8 for $M_{\text {clear }}$, with $n=1 \mathrm{~cm}^{-3}$, to investigate how the implementation of the metallicity dependence affects our results.

\section{RESULTS}

Figure 1 shows the evolution of the DTM ratio for models with a constant $M_{\text {clear }}$, with $y_{\text {dust }}$ varied between the observationally-favoured 0.05 , and the 0.001 determined from previous work on the DTM ratio (e.g. Galliano et al. $2021)^{2} . f_{\text {acc }}$ is chosen to reproduce the observed rise in DTM ratio between 0.1 and $0.5 \mathrm{Z}_{\odot}$, and the value of $\sim 0.5$ at $Z_{\text {tot }}=Z_{\odot}$. At low values of $Z_{\text {tot }}$, the efficiency of both destruction and growth in the ISM is low, and the DTM ratio tends towards the intrinsic value from stellar production. Reproducing the low values seen at $Z_{\text {tot }} \sim 0.1 \mathrm{Z}_{\odot}$ then requires $y_{\text {dust }} \lesssim 0.01$, which corresponds to a dust yield per CCSN of $\lesssim 0.1 \mathrm{M}_{\odot}$, as found by Galliano et al. (2021) and other previous studies.

The left panel of Figure 1 shows the impact of a metallicity-dependent $M_{\text {clear }}$, implemented according to Equation 8. The increased dust destruction at low metallicity counteracts the high stellar dust yields, so that the $\mathrm{DTM}$ ratio at $0.1 \mathrm{Z}_{\odot}$ is significantly lower than the intrinsic stellar value. An observed value of $Z_{\text {dust }} / Z_{\text {tot }} \sim 0.01$ is then consistent with an average CCSN yield of $0.1 \mathrm{M}_{\odot}$, and the low- $Z$ data as a whole do not rule out the $\sim 0.5 \mathrm{M}_{\odot}$ dust masses seen in supernova remnants. Using instead the Y11 prescription for $M_{\text {clear }}$, shown in the right panel of Figure 1, the weaker metallicity dependence results in a more modest decrease in the low- $Z$ DTM ratio compared to the constant$M_{\text {clear }}$ models, but the observed DTM ratios of $\lesssim 0.1$ can still be reproduced by models with substantial $\left(\gtrsim 0.1 \mathrm{M}_{\odot}\right.$ per CCSN) stellar dust yields.

\section{DISCUSSION \& CONCLUSIONS}

It is often claimed that the low DTM ratios seen in some

2 The dust yield per CCSN, including any AGB-formed dust, is $y_{\text {dust }} \times f_{\text {ret }} / f_{\mathrm{SN}}=10 y_{\text {dust }} \mathrm{M}_{\odot}$, so these values correspond to 0.5 and $0.01 \mathrm{M}_{\odot}$ per CCSN respectively. 
galaxies require similarly low dust yields from CCSNe. We have shown that this interpretation is affected by the assumption of a constant dust destruction efficiency. If preexisting dust grains are more easily destroyed in lowermetallicity gas, and the resulting increase in destruction efficiency depends strongly enough on metallicity, there is no tension between the large dust masses found in supernova remnants and the relatively small dust masses in lowmetallicity galaxies.

As we note in Appendix A, Equation 8 is likely to overestimate the strength of the metallicity dependence of $M_{\text {clear }}$ by neglecting kinetic sputtering. This explains the weaker dependence found by Y11, who include this process. However, Y11 themselves neglect grain shattering, which can have an enormous impact on dust destruction rates (Kirchschlager et al. 2019). While shattering should not be greatly affected by the gas-phase metallicity, we would expect it to redistribute mass from large to small grains, and therefore increase the importance of thermal compared to kinetic sputtering ${ }^{3}$. A more complete treatment of dust destruction as a function of metallicity may therefore be closer to the scaling in Equation 8 than to the Y11 results.

A limitation of our model (and most other single-phase models) is the assumption that newly-produced metals and dust are instantaneously mixed with the entire gas reservoir. As dust production is likely to occur in the vicinity of star-forming regions, either due to stellar dust production or growth in dense molecular gas, the ISM swept up by CCSNe is likely to be more dust-enriched than average. Equation 7 will then underestimate the actual rate of dust destruction. This effect is particularly severe at low metallicity, where the instantaneous-mixing assumption results in such a low $Z_{\text {dust }}$ that destruction by $\mathrm{SNe}$ is negligible, and DTM ratios increase from $Z_{\text {tot }} \sim 0.01 \mathrm{Z}_{\odot}$ to $Z_{\text {tot }}=0$ (Figure 1 ). As such, we may still be underestimating dust destruction at low metallicity. On the other hand, low-metallicity dwarf galaxies are only found to be chemically inhomogeneous by factors of a few (Lebouteiller et al. 2009, 2013; James et al. 2016, 2020), so mixing may be efficient enough to limit the importance of this effect.

A metallicity-dependent dust destruction efficiency is far from the only possible cause of an increase in the DTM ratio. While we have assumed stellar dust yields are constant, as indicated by models, the current theoretical understanding of dust formation appears to be incomplete (Wesson et al. 2015; Bevan \& Barlow 2016; Priestley et al. 2020) so this may well be inaccurate. De Vis et al. (2021) have recently reconciled high stellar dust yields with low DTM ratios by invoking photofragmentation, although as this process only affects carbon grains, it is unclear whether it can achieve the required/reduction in dust mass. As our proposed mechanism is based on comparatively wellunderstood physical processes, which must be in operation to some extent in the ISM, we regard it as a more promising explanation of the observational data.

By allowing high stellar dust yields without an increase

3 Large grains, due to their high inertia, are more strongly affected by kinetic sputtering, whereas small grains are typically well-coupled to the gas velocity but rapidly eroded by hot gas due to their higher surface area to volume ratios. in the destruction rate, at least at higher metallicities, an evolving $M_{\text {clear }}$ inevitably results in a greater importance for stellar dust production compared to ISM growth. With $M_{\text {clear }}$ given by Equation 8, the lowest observed DTM ratios can be reproduced with a value of $y_{\text {dust }}$ an order of magnitude larger than that required by a constant $M_{\text {clear }}$ model. This results in a corresponding order of magnitude increase in the contribution of stellar sources to the overall dust budget. Depending on the values of the other parameters in our model, this increase may not be of huge importance - the majority of the dust mass after $10 \mathrm{Gyr}$ is still ISM-grown for our models, because the adopted value (or normalisation) of $M_{\text {clear }}$ results in CCSNe destroying more dust than is formed in stars by the time $Z_{\text {tot }} \sim \mathrm{Z}_{\odot}$. However, the common assertion that stellar dust production is unimportant compared to grain growth, in both low- and high-redshift galaxies, may be reliant on the assumption of a constant destruction efficiency. We suggest that models making this assumption are reevaluated in order to determine its impact.

\section{ACKNOWLEDGEMENTS}

FDP is funded by the Science and Technology Facilities Council. IDL acknowledges support from European Research Council (ERC) starting grant 851622 DustOrigin. MJB acknowledges support from the ERC grant SNDUST ERC-2015-AdG-694520.

\section{DATA AVAILABILITY}

The data underlying this article will be made available upon request. A Fortran implementation of the model is available at ww. github.com/fpriestley/galaxy.

\section{REFERENCES}

Asplund M., Grevesse N., Sauval A. J., Scott P., 2009, ARA\&A, 47,481

Bevan A., Barlow M. J., 2016, MNRAS, 456, 1269

Biscaro C., Cherchneff I., 2016, A\&A, 589, A132

Chawner H., et al., 2019, MNRAS, 483, 70

Chawner H., et al., 2020, MNRAS, 499, 5665

Cristallo S., Straniero O., Gallino R., Piersanti L., Domínguez I., Lederer M. T., 2009, ApJ, 696, 797

Dame T. M., 1993, in Holt S. S., Verter F., eds, American Institute of Physics Conference Series Vol. 278, Back to the Galaxy. pp 267-278, doi:10.1063/1.43985

De Looze I., Barlow M. J., Swinyard B. M., Rho J., Gomez H. L., Matsuura M., Wesson R., 2017, MNRAS, 465, 3309

De Looze I., et al., 2019, MNRAS, 488, 164

De Looze I., et al., 2020, MNRAS, 496, 3668

De Vis P., et al., 2017, MNRAS, 471, 1743

De Vis P., et al., 2019, A\&A, 623, A5

De Vis P., Maddox S. J., Gomez H. L., Jones A. P., Dunne L., 2021, MNRAS, 505, 3228

Ferrara A., Peroux C., 2021, MNRAS, 503, 4537

Ferrara A., Viti S., Ceccarelli C., 2016, MNRAS, 463, L112

Galliano F., et al., 2021, A\&A, 649, A18

Hu C.-Y., Zhukovska S., Somerville R. S., Naab T., 2019, MNRAS, 487, 3252

James B. L., Auger M., Aloisi A., Calzetti D., Kewley L., 2016, ApJ, 816, 40 
James B. L., Kumari N., Emerick A., Koposov S. E., McQuinn K. B. W., Stark D. P., Belokurov V., Maiolino R., 2020, MNRAS, 495, 2564

Jones A. P., Tielens A. G. G. M., Hollenbach D. J., McKee C. F., 1994, ApJ, 433, 797

Kirchschlager F., Schmidt F. D., Barlow M. J., Fogerty E. L., Bevan A., Priestley F. D., 2019, MNRAS, 489, 4465

Lebouteiller V., Kunth D., Thuan T. X., Désert J. M., 2009, A\&A, 494,915

Lebouteiller V., Heap S., Hubeny I., Kunth D., 2013, A\&A, 553, A16

Limongi M., Chieffi A., 2018, ApJS, 237, 13

Marassi S., Schneider R., Limongi M., Chieffi A., Graziani L., Bianchi S., 2019, MNRAS, 484, 2587

Martínez-González S., Wünsch R., Silich S., Tenorio-Tagle G., Palouš J., Ferrara A., 2019, ApJ, 887, 198

Mathis J. S., Rumpl W., Nordsieck K. H., 1977, ApJ, 217, 425

Matsuura M., et al., 2015, ApJ, 800, 50

Mattsson L., Andersen A. C., Munkhammar J. D., 2012, MNRAS, 423, 26

Morgan H. L., Edmunds M. G., 2003, MNRAS, 343, 427

Nanni A., Bressan A., Marigo P., Girardi L., 2013, MNRAS, 434,2390

Nanni A., Burgarella D., Theulé P., Côté B., Hirashita H., 2020, A\&A, 641, A168

Niculescu-Duvaz M., Barlow M. J., Bevan A., Milisavljevic D., De Looze I., 2021, MNRAS, 504, 2133

Priestley F. D., Barlow M. J., De Looze I., 2019, MNRAS, 485,440

Priestley F. D., Bevan A., Barlow M. J., De Looze I., 2020, MNRAS, 497, 2227

Priestley F. D., Chawner H., Matsuura M., De Looze I., Barlow M. J., Gomez H. L., 2021, MNRAS, 500, 2543

Robitaille T. P., Whitney B. A., 2010, ApJ, 710, L11

Salpeter E. E., 1955, ApJ, 121, 161

Sutherland R. S., Dopita M. A., 2017, ApJS, 229, 34

Triani D. P., Sinha M., Croton D. J., Pacifici C., Dwek E., 2020, MNRAS, 493, 2490

Wesson R., Barlow M. J., Matsuura M., Ercolano B., 2015, MNRAS, 446, 2089

Woosley S. E., Weaver T. A., 1995, ApJS, 101, 181

Yamasawa D., Habe A., Kozasa T., Nozawa T., Hirashita H. Umeda H., Nomoto K., 2011, ApJ, 735, 44

\section{APPENDIX A: THE METALLICITY SCALING OF DUST DESTRUCTION}

We calculate the evolution of plane-parallel shocks in a $1 \mathrm{~cm}^{-3}$ ambient medium of varying metallicity using MAPPINGS (Sutherland \& Dopita 2017). We use the gas density and temperature (as a function of time post-shock) to calculate the thermal sputtering rate, $\frac{d a}{d t}$, of silicate grains following the prescription in Biscaro \& Cherchneff (2016). We then integrate $\frac{d a}{d t}$ from $t=0$, when the shock first impacts the ambient medium, to the time where the gas has cooled below $10^{4} \mathrm{~K}$. This gives the reduction in grain size, $a_{\text {sput }}$, due to thermal sputtering in the shocked gas. We determine the destruction efficiency, $f_{\text {dest }}$, by taking a Mathis et al. (1977) $\frac{d n}{d a} \propto a^{-3.5}$ grain size distribution and calculating the reduction in dust mass if every grain has its size reduced by

Figure A1 shows the variation of $f_{\text {dest }}$ with metallicity for a $200 \mathrm{~km} \mathrm{~s}^{-1}$ shock impacting a $1 \mathrm{~cm}^{-3}$ ambient medium. The results are well-fit by $f_{\text {dest }}=0.64 /\left(1+Z / 0.14 \mathrm{Z}_{\odot}\right)$. We interpret the $f_{\text {dest }} \sim Z^{-1}$ behaviour at high $Z$ as due to the

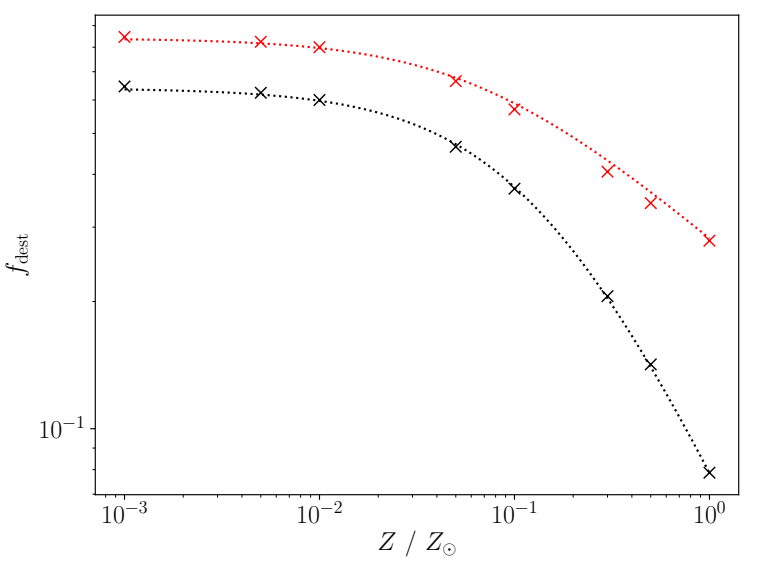

Figure A1. The dust destruction efficiency via thermal sputtering for a $200 \mathrm{~km} \mathrm{~s}^{-1}$ shock versus metallicity (black crosses), and with an additional efficiency of 0.2 to represent kinetic sputtering (red crosses). The thermal model efficiencies are well-reproduced by $f_{\text {dest }}=0.64 /\left(1+Z / 0.14 \mathrm{Z}_{\odot}\right)$ (black dotted line $)$, the increased values by $f_{\text {dest }}=0.84 /\left(1+Z / 0.07 \mathrm{Z}_{\odot}\right)^{0.4}$ (red dotted line).

approximately linear relationship between the cooling rate and the metallicity for temperatures $\gtrsim 10^{5} \mathrm{~K}$, where cooling is dominated by metal line emission. The cooling timescale is then $t_{\text {cool }} \sim Z^{-1}$, so the period of time where thermal sputtering is effective also varies as $Z^{-1}$, and if $\frac{d a}{d t} \sim$ const. then $a_{\text {sput }} \approx Z^{-1}$ (we have confirmed that this is in fact the case in our implementation). Assuming a single grain size $a_{0}$ for simplicity, $f_{\text {dest }}=1-\left(a_{0}-a_{\text {sput }}\right)^{3} / a_{0}^{3}$ which, for $a_{\text {sput }} \ll$ $a_{0}$, reduces to $f_{\text {dest }} \sim 3 a_{\text {sput }} / a_{0}$. As the majority of the mass in a Mathis et al. (1977) distribution is in the largest grains, where $a_{\text {sput }} \ll a_{0}$ holds (at least for a $200 \mathrm{~km} \mathrm{~s}^{-1}$ shock), $f_{\text {dest }} \sim Z^{-1}$ until the point where metal line cooling becomes unimportant, beyond which $f_{\text {dest }} \sim$ const..

Obtaining the mass of dust destroyed per SN from the dust destruction efficiency of single-shock models requires assumptions about the typical evolution of supernova remnants and their surrounding ISM, which may not accurately describe the complex behaviour of real objects (Chawner et al. 2020; Priestley et al. 2021). Rather than attempt to derive a value of $M_{\text {clear }}$ from $f_{\text {dest }}$, we treat the normalisation of $M_{\text {clear }}$ (the value at some reference metallicity) as a free parameter, and assume that it follows the same scaling with $Z$ as $f_{\text {dest }}\left(\right.$ i.e. $\left.M_{\text {clear }} \propto\left(1+Z / 0.14 \mathrm{Z}_{\odot}\right)^{-1}\right)$. Because the physical process causing this scaling - the increase in the cooling timescale with decreasing $Z$ - is only weakly affected by the shock velocity and ambient density, we expect that it should be preserved regardless of how a value of $M_{\text {clear }}$ is obtained from $f_{\text {dest }}$.

Our model neglects kinetic sputtering, which does not have any obvious dependence on metallicity. If we add a constant value of 0.2 to $f_{\text {dest }}$ at all metallicities to approximate this effect, shown in Figure A1, we find a much shallower $f_{\text {dest }} \sim Z^{-0.4}$, close to the -0.298 exponent from Y11. The $f_{\text {dest }} \sim Z^{-1}$ relation derived from thermal sputtering may thus represent an extreme case, which we use in this paper to assess the potential impact, although we argue in Section 4 that grain shattering (not included by Y11) might result in a stronger metallicity dependence than sputtering alone. 